\title{
Nonlinear Dynamical Screening Effects and strong Local Fluctuations of Drag Forces in Collective Scattering of Particle Streams on Impurity Ensembles ${ }^{\dagger}$
}

\author{
Oleksandr Kliushnichenko and Sergey Lukyanets \\ Institute of Physics, National Academy of Science of Ukraine, Kyiv, Ukraine \\ + Presented at the Entropy 2021: The Scientific Tool of the 21st Century, 5-7 May 2021; Available online: \\ https://sciforum.net/conference/Entropy2021/.
}

Published: 5 May 2021

We study the effects of nonequilibrium correlations and interactions between constituent particles of a bunch or pulsed beam, arising in course of its motion through a medium, or under the scattering of particle stream on a cluster or finite cloud of impurities [1]. Formally, these correlations are determined by the effect of dynamical screening. Such induced correlations and dynamical friction forces on impurities are manifested most pronouncedly in the case of collective dynamical screening effect and are enhanced in the case of a nonlinear medium when strong local fluctuations of scattered field begin to act as additional scattering elements along with impurities. In addition, collective scattering effects depend on the degree of impurity cluster disorder [2]. We focus on effects provoked by the collective scattering on randomly inhomogeneous structures and by the presence of local fluctuations. The presence of strong fluctuations of the scattered field is shown to give rise to strong local fluctuations of nonequilibrium forces acting on certain particles within the impurity cluster that can be a precursor of dynamical instability of the cluster, which is manifested in the peculiar behavior of the tails of probability distribution function for the drag force [3]. The description of the impurity cluster in terms of effective parameters breaks down due to the presence of such fluctuations.

Acknowledgments: This work was partially supported by a grant for research groups of young scientists from the National Academy of Science of Ukraine (Project No. 0120U100155).

\section{References}

1. Kliushnychenko, O.V.; Lukyanets, S.P. Effects of gas interparticle interaction on dissipative wake-mediated forces. Phys. Rev. E 2017, 95, 012150.

2. Kliushnychenko, O.V.; Lukyanets, S.P. Effects of collectively induced scattering of gas stream by impurity ensembles: Shock-wave enhancement and disorder-stimulated nonlinear screening. Phys. Rev. E 2018, 98, 020101.

3. Kliushnichenko, O.V.; Lukyanets, S.P. Collective wake-mediated interactions in lattice fluids: Effects of strong local force fluctuations stimulated by impurity disorder. arXiv 2020, arXiv:2012.09266.

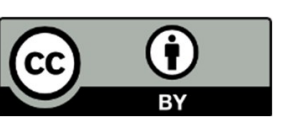

(C) 2021 by the authors. Licensee MDPI, Basel, Switzerland. This article is an open access article distributed under the terms and conditions of the Creative Commons Attribution (CC BY) license (http://creativecommons.org/licenses/by/4.0/). 